\title{
Análisis técnico, socioeconómico y ambiental de la electrificación con energía solar fotovoltaica aislada para vivienda rural en Hato Corozal, Casanare, Colombia
}

\author{
Technical analysis, socio-economic and \\ environmental of the electrification with \\ photovoltaics isolated for rural housing \\ in Hato Corozal, Casanare, Colombia
}

\author{
Análise técnica, socioeconômica e ambiental da \\ electrificação solar fotovoltaica para a habitação \\ rural isolada em Hato Corozal, Casanare, Colombia
}

\author{
Edwin Blasnilo Rúa Ramírez ${ }^{1}$, Andrea Isabel Barrera Siabato², Martín Gómez Orduz ${ }^{3}$ \\ 1'Ingeniero Mecánico, Master Oficial de Investigación en Ingeniería Termodinámica de Fluidos, \\ Magister en Gerencia de Empresas mención Industria, Máster en Ingeniería de Climatización de Edificios, \\ Candidato a Doctor en Proyectos en la Universidad Iberoamericana de México. ${ }^{2}$ Ingeniera Industrial, \\ Especialista en Salud Ocupacional, Magister en Dirección y Administración de Empresas. \\ ${ }^{3}$ Licenciado en Física y Matemáticas, Especialista en Educación Superior a Distancia, Master Online Education. \\ 1,2,3 Escuela de Ciencias Básicas, Tecnología e Ingenierías -ECBTI. \\ Universidad Nacional Abierta y a Distancia UNAD. Yopal, Casanare. Colombia.
}

1edwin.rua@unad.edu.co; 2andreai.barrera@unad.edu.co; ${ }^{3}$ martin.gomez@unad.edu.co

\section{Resumen}

La electrificación con energía solar fotovoltaica aislada es la opción más viable para vivienda rural, en especial si se encuentra muy distante del sistema nacional interconectado. Hato Corozal, Casanare, Colombia, es un municipio estructurado en su área rural por fincas muy alejadas entre sí. Su mayor inconveniente de desarrollo social y económico es la falta de electricidad como servicio de primera necesidad. La gobernación de Casanare a través del fondo nacional de regalías aprobó la instalación de 206 sistemas solares fotovoltaicos (SFV) a las familias más alejadas y más vulnerables del municipio. El objetivo principal de esta investigación fue realizar una evaluación socio-económica de las familias de la zona rural del municipio, beneficiadas con la instalación de estos sistemas SFV. Además, se realizó un análisis técnico de simulación de la operación del SFV dimensionado con 2 días sin sol y luego 12 días con sol, también se hizo una simulación de la operación del SFV dimensionado con 14 días con sol. Esta investigación se abordó desde el enfoque metodológico descriptivo y de investigación aplicada de campo que incluyó visita a las viviendas, aplicación, análisis de instrumentos y registro fotográfico. Los resultados presentan gran incidencia desde un enfoque social, económico y ambiental que puede llegar a ser implementado en otras áreas rurales del país como ejemplo de uso de tecnologías limpias en favor del desarrollo rural sostenible.

Palabras clave: Energía Solar Fotovoltaica, redes cableado, electrificación rural. 


\section{Abstract}

The electrification with solar PV isolation is the most viable option for rural housing, especially if it is very distant from the national interconnected system. Hato Corozal, Casanare, Colombia, is a municipality structured in its rural area by farms very far away from each other. Its major drawback of social and economic development is the lack of electricity as a service of first necessity. Casanare governorate through the royalties national fund approved the installation of 206 solar photovoltaic systems (SFV) from families farthest and most vulnerable of the municipality. The main objective of this research was to conduct a socio-economic evaluation of the families in the rural area of the municipality, benefited with the installation of these systems SFV. In addition, a technical analysis of simulation of the operation of the SFV dimensioned 2 days without Sun and then 12 days with Sun, also did a simulation of the operation of the SFV dimensioned with 14 days with Sun. This research was addressed from the descriptive methodological approach and applied field research which included visits to homes, application, analysis instruments and photographic record. The results have great incidence from a social, economic and environmental approach that can be implemented in other rural areas of the country as an example of the use of clean technologies in favor of sustainable rural development.

Key-words: Photovoltaic Solar Energy, networks wiring, rural electrification.

\section{Resumo}

A eletrificação com energia solar fotovoltaica isolada é a opção mais viável para a moradia rural, especialmente se o local encontra-se afastado do sistema nacional interconectado. Hato Corozal, Casanare, Colômbia, é uma localidade estruturada na área rural por fazendas afastadas entre elas. Seu maior problema de desenvolvimento social e econômico é a falta de energia como serviço básico. A governação de Casanare, através do fundo nacional de regalias aprovou a instalação de 206 sistemas solares fotovoltaicos (SFV) às famílias mais afastadas e vulneráveis da localidade. O objetivo principal deste trabalho de pesquisa foi realizar uma avaliação socioeconômica das famílias da zona rural da localidade, beneficiadas com a instalação dos sistemas SVF. Além disso, foi realizada uma análise técnica de simulação da operação do SFV dimensionado com 2 dias sem sol e logo após foi feita uma simulação da operação do SFV dimensionado com 14 dias com sol. Esta pesquisa, teve uma abordagem metodológica descritiva e de pesquisa aplicada de campo que incluía visitas às moradias, aplicação, análises de instrumentação e registro fotográfico. Os resultados mostram uma grande incidência desde a parte social, econômica e ambiental, que pode ser usada em outras áreas rurais do país, por exemplo, o uso de tecnologias limpas que favoreçam o desenvolvimento sustentável.

Palavras-chave: Energia Solar Fotovoltaica, redes a cabo, eletrificação rural.

\section{Introducción}

Dentro de la nueva agenda para el desarrollo sostenible propuesta en 2012 por la Organización de las Naciones Unidas (ONU), se plantean 17 objetivos (Objetivos de Desarrollo Sostenible ODS) entre los cuales la meta 7 es precisamente garantizar el acceso a energía asequible, segura, sostenible y moderna para todos (PNUD, 2012). El logro de este objetivo es particularmente relevante en países en vías de desarrollo y especialmente en comunidades rurales aisladas, pues la falta de provisión de fuentes de energía modernas, amplía las brechas de desigualdad social, cultural, ambiental y económica de manera dramática. Iniciativas como The Sustainable Energy for All (IEA, UNDP, UNIDO (s.f.); Modi et al., 2005) y la declaración por parte de la asamblea de las Naciones Unidas de la década 2014-2024 como la década de la energía sostenible demuestran la importancia del tema en la agenda de todos los países del planeta (Aguer et al., 2004). 
La electricidad, vector energético que es el foco de esta evaluación, es considerada una de las fuentes de energía moderna que ha permitido un mayor avance en los indicadores de desarrollo humano (IDH), y de hecho existe una fuerte correlación entre la una y la otra (CEPAL ONU, 2009), a menores consumos de energía provenientes de fuentes modernas, se acompañan invariablemente de menores IDH. No obstante, el sólo acceso a esta fuente de energía no garantiza por sí sola un impacto en términos de mejora en calidad de vida, ingresos, o reducción de pobreza en particular en las zonas rurales (Cherny, 2004). La electricidad no debe representar un fin en sí mismo, y por tanto el enfoque de cobertura del servicio de los gobiernos debe ser urgentemente redefinido (Garcés, 2013).

En el llamado trilema de la sostenibilidad energética planteado por el Concejo Mundial de la Energía -WET por sus siglas en inglés- se involucran: la seguridad energética en términos de satisfacer la demanda de energía actual y futura en adecuada cantidad y calidad; la equidad social en cuanto a la accesibilidad y asequibilidad del suministro de energía para toda la población; y la sostenibilidad ambiental tanto del suministro como de la demanda, así como la inclusión de oferta renovable (WET, 2012).

De esto se desprende que la universalización del servicio de energía eléctrica debe ser analizada y evaluada en un contexto amplio de elementos fuertemente interrelacionados en los cuales características sociales, culturales, económicas, ambientales y geográficas condicionarán la oferta y el uso de la energía, los requerimientos de cantidad y la sostenibilidad de la canasta energética disponible para cada caso particular.

Según el Plan Indicativo de Expansión y Cobertura 2013-2017 PIEC (Sánchez, 2010) elaborado por la Unidad de Planeación Minero Energética -UPME- del Ministerio de Minas y Energía, se encuentra que para el caso de Colombia el Índice de Cobertura de Energía Eléctrica -ICEE- al 2012 era del $96,1 \%$, es decir que según este censo 470.244 viviendas no cuentan con servicio de energía eléctrica ( $3,9 \%$ del total nacional), de las cuales se estima que el $91,98 \%$, que representan alrededor de 432.530 viviendas, están ubicadas fuera de las cabeceras municipales, es decir en zonas rurales y que de éstas aproximadamente 55.809 viviendas $(0,46 \%$ del total nacional) no son interconectables al sistema eléctrico nacional -SIN-, es decir requieren soluciones aisladas.

Hato Corozal, Casanare, tiene una extensión de $5.518 \mathrm{~km}^{2}$ y una población aproximada de 11.500 habitantes. Se encuentra a una altitud de $250 \mathrm{msnm}$, con temperaturas de 25 a $27^{\circ} \mathrm{C}$. La extensión área urbana es de $1.6 \mathrm{~km}^{2}$ y la extensión área rural es de $5.516 .59 \mathrm{~km}^{2}$. La mayor parte de la población rural presenta altos índices de Necesidades Básicas Insatisfechas -NBI-, no cuentan con energía eléctrica, ni servicios como agua potable y alcantarillado, hay un alto déficit de infraestructura como vías, redes de distribución y comunicaciones (Miguel, 2014).

En este contexto las soluciones de energía solar fotovoltaica emergen como una alternativa válida desde la perspectiva del trilema energético planteado por la WEC, y es así como la gobernación de Casanare pensando en mejorar la calidad de vida de la población rural, a través del Fondo Nacional de Regalías, aprobó recursos para la Instalación de 206 sistemas de energía alternativa solar fotovoltaicas para viviendas rurales del municipio de Hato Corozal en zonas no interconectadas (Concejo municipal de Yopal, 2012: Empresa de Energía de Casanare, 2003).

\section{Materiales y métodos}

Se realizó una investigación de tipo descriptivo e investigación aplicada de campo, se valoró la instalación de doscientos seis (206) sistemas solares fotovoltaicos en viviendas rurales aisladas del municipio de Hato Corozal, Casanare. La información fue recolectada a través de fuentes primarias: medición, observación directa, encuestas, cuestionarios, entrevistas a los beneficiados, y fuentes secundarias: bibliográficas y documentos gubernamentales provenientes de la Cámara de comercio, Consorcio Hato Solar. 
El trabajo de campo incluyó visitas a las viviendas favorecidas, registro fotográfico, georreferenciación con equipo GPS y la aplicación de un instrumento cuestionario - encuesta tipo escalamiento Likert. La validación del cuestionario - encuesta se hizo a través de prueba piloto y validación por expertos. Para la determinación del tamaño de la muestra se emplearon características del muestreo aleatorio simple para poblaciones finitas, dado que este tipo de muestreo permite determinar el número de encuestas teniendo en cuenta los siguientes aspectos: Cálculo del tamaño de muestra empleando un 95\% de confianza y un margen de error del $5 \%$, distribución proporcional, de acuerdo a los predios objeto de estudio, con el objeto de tener una muestra con características lo suficientemente representativas para el estudio. Con base en lo anterior se definió muestrear 132 viviendas rurales.

Para el análisis estadístico de datos se aplicó el análisis de varias variables, estadística descriptiva y muestreo. Se Analizaron la interrelación entre los valores de variables nominales según distribución de casos. Se utilizó el software estadístico SPSS (StatisticalPackageforthe Social Sciences) versión 23.0 para el análisis de los datos.

\section{Resultados y discusión}

Los resultados se abordan desde el enfoque técnico con la evaluación del sistema y posteriormente se presenta el análisis respectivo a partir del instrumento empleado en la recolección de la información.

\section{Análisis Técnico}

La selección o escogencia del suministro de energía a través de tecnología solar fotovoltaica se realizó luego de un exhaustivo análisis que comprendió aspectos como la cantidad de energía demandada, evaluación de la disponibilidad del recurso y el desempeño mínimo esperado. Desde el enfoque de consumo, la base de partida para un correcto programa de energización rural es la comprensión de las necesidades de los usuarios que van a emplear la fuente de energía, es decir los usos que van a darse para el mejoramiento de su calidad de vida (IDH), con el aumento o mejora de actividades o procesos que les permitan obtener ingresos y mejorar su productividad.

En la resolución 355 de 2004 de la UPME, se define como consumo de subsistencia, la cantidad mínima de electricidad utilizada en un mes por un usuario típico para satisfacer las necesidades básicas que solamente puedan ser satisfechas mediante esta forma de energía final. Se establece el Consumo de Subsistencia en $173 \mathrm{kWh}$-mes para alturas inferiores a $1.000 \mathrm{msnm}$ (Ministerio de Minas y Energía, 2013). Esto para el servicio prestado por redes físicas, según lo establecido en el artículo 8 de la ley 632 del 2000 (Domenech, 2013).

Sin embargo, según el PIEC 2014-2017 (Ministerio de Minas y Energía, 2013) se considera un consumo promedio mensual de energía eléctrica para usuarios residenciales en áreas rurales del sistema interconectado nacional del orden de los $92 \mathrm{kWh}$ mes. Con lo que se ajusta a un valor más real de la demanda que los usuarios de áreas rurales requieren de esta fuente de energía. Al revisar las cifras reportadas por la empresa prestadora del servicio eléctrico en el departamento de Casanare, se encuentra que el consumo de energía eléctrica promedio para viviendas de estrato 1 es de $80 \mathrm{kWh}$ mes, lo que equivale a un consumo promedio de 2,67 kWh-día (Empresa de Energía del Casanare, 2003). Se considera que estos $80 \mathrm{kWh}$-mes son la cantidad de energía eléctrica mínima que debe ser garantizada por la fuente que provea la solución a los usuarios rurales dispersos en el municipio de Hato Corozal. Ahora bien, frente al análisis de la oferta y la demanda, Para abastecer esta demanda de energía se tienen diferentes alternativas: la primera es llevar el servicio extendiendo las redes físicas del sistema interconectado, la segunda es con equipos electrógenos, plantas diésel o gasolina, y como tercera alternativa a través de soluciones solares fotovoltaicas aisladas.

Cuando se presentan situaciones de alta dispersión de los beneficiarios la opción de extender redes se 
hace demasiado costosa, y si a este factor se agrega el de baja demanda de energía el resultado es prácticamente inviable cualquier proyecto por los altos costos que se erogan de este tipo de proyectos siendo entonces las soluciones fotovoltaicas una opción atractiva gracias a los bajos costos de instalación (Gasquet, 2004).
Con el fin de dimensionar el desempeño del sistema frente a las evaluaciones económica y Financiera la Tabla 1 presenta un comparativo de costos de inversión del Sistema de Interconexión Eléctrica Nacional SIN versus El Sistema Fotovoltaico SFV para suministro de energía eléctrica a una vivienda rural de estrato 1 en el departamento de Casanare.

Tabla 1. Comparativo costos de inversión SIN Vs SFV

\begin{tabular}{l|c|c|c}
\hline & \multicolumn{2}{c}{ SIN } & S. FV. \\
& $\begin{array}{c}\text { Red Rural } \\
13,2 \mathrm{KV}\end{array}$ & $\begin{array}{c}\text { Red Rural } \\
\text { baja tensión }\end{array}$ & Aislado \\
\hline Costo de inversión & $\$ 22,351,780$ & $\$ 21.247 .050$ & $\$ 19,991,700$ \\
\hline Distancia a vivienda & $750 \mathrm{~m}$ & $600 \mathrm{~m}$ & $10 \mathrm{~m}$ \\
\hline
\end{tabular}

Fuente: Empresa de Energía del Casanare, (2003).

Se observa que es más económico invertir en el SFV que en el SIN para suministrar electricidad a una vivienda rural estrato 1 en Casanare, cuando esté a más de 750 metros de la red de interconexión en nivel de tensión $2(13,2$ KV 2Ø) o esté a más de 600 metros de la red de interconexión en nivel de tensión 1 (1Ø - $3 \mathrm{H}$ - baja tensión).
Con respecto a la verificación del correcto funcionamiento del SFV se analizaron dos simulaciones para observar su desempeño con 14 días soleados, y con dos días sin sol y luego doce días soleados. En las Figuras 1 y 2, se observa el desempeño del SFV en las dos condiciones analizadas.

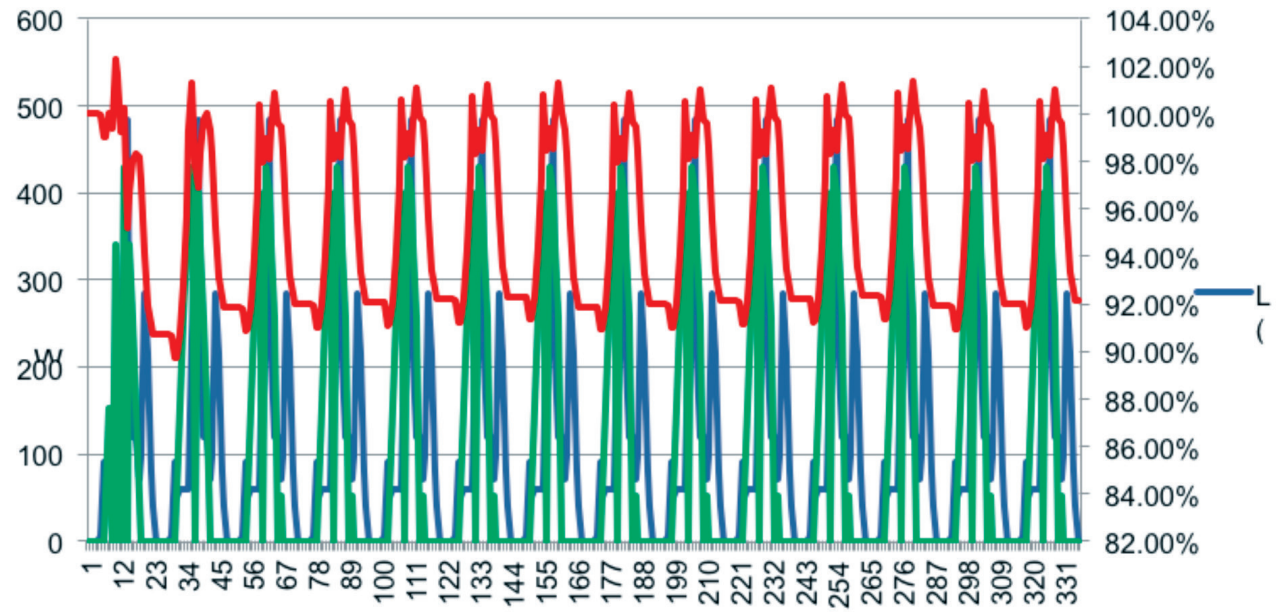

Figura 1. Simulación de la operación del SFV dimensionado con 14 días con sol

Dónde: $L$ es el consumo energético horario de la vivienda rural estrato 1 en Wh. EFV es la energía suministrada directamente a la carga por el sistema fotovoltaico en Wh. SOC es el estado de carga del banco de baterías. 
En la Figura 1 se observa cómo el banco de baterías mantiene diariamente un estado de carga mínimo del $89 \%$ cuando todos los días son soleados, atendiendo todo el día el servicio de energía eléctrica demandado por la vivienda con el SFV con acumulación diseñado, con una profundidad de descarga máxima diaria del banco de baterías del $11 \%$.

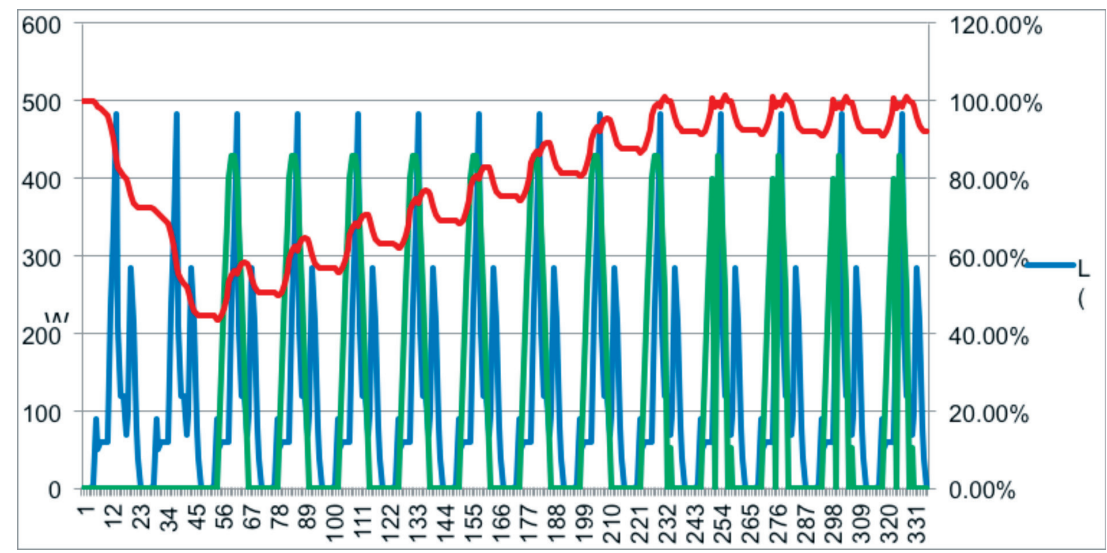

Figura 2. Simulación de la operación del SFV dimensionado con 2 días sin sol y luego 12 días con sol.

En la Figura 2 se observa cómo el banco de baterías atiende diariamente durante dos días sin sol el servicio de energía eléctrica demandado por la vivienda, con una profundidad de descarga máxima del banco de baterías del $56 \%$-estado de carga mínima del 44\%-, garantizando una autonomía de dos días alimentando la carga de la vivienda, sin que las baterías sufran una profundidad de descarga superior al $60 \%$. De la gráfica también se observa que luego de los dos días sin sol, al tener sol durante doce días, el banco de baterías va recuperando diariamente su estado de carga hasta llegar a un valor mínimo del 85\% -profundidad de descarga del 15\%), atendiendo el servicio de energía eléctrica demandado por la instalación.

\section{Análisis Socioeconómico:}

Para la evaluación socioeconómica se aplicó el instrumento - cuestionario a una muestra de 132 viviendas, según lo calculado para una población total de 206 usuarios beneficiados, las características principales y sus respectivos resultados se muestran a en las Figuras 3 a 10.
Frente al material de construcción de la vivienda un $93 \%$ de los usuarios fabricaron sus viviendas en Bloque o ladrillo, solo un $1 \%$ construyeron sus hogares en Zinc para un total de 3 usuarios (Figura 3).

Para el caso del material de los pisos de las viviendas analizadas el de menor uso es el de Baldosa con 3 ítems, el de mayor uso corresponde a Cemento, seguido por el de tierra (Figura 4).

Frente al material en que está construido el techo los principales corresponden a Zinc y Palma, los de menor índice corresponden a teja eternit u otro material el cual no se especifica (Figura 5).

Aproximadamente en el $37.1 \%$ de los predios viven más de 4 personas, solo en 18 (8.6\%) Hogares de los encuestados habitan 2 personas (Figura 6).

El $93 \%$ de los habitantes obtienen el agua de pozo profundo, con motobomba a base de combustible. La electrificación con S. Fv. Les permite instalar electrobombas y así reducir el consumo de combustibles. Lo que también les trae un ahorro económico mensual (Figura 7). 
Aproximadamente el $67 \%$ de los habitantes cuentan con baño a pozo séptico, el $30 \%$ no tienen sanitario y el 3\% tienen inodoro sin conexión o letrina (Figura 8).

El $83 \%$ de las viviendas utilizan velas y linternas de pilas para iluminar. El restante utiliza lámparas de gasolina y otros medios. Este proyecto les permitirá mejorar la iluminación de la vivienda con bombillos leds y evitar el consumo de combustibles y velas (Figura 9).

Se identifica que las viviendas objeto del estudio eliminan las basuras a través de la quema y en último lugar mediante la recolección de las mismas (Figura 10).

Los resultados de las encuestas demostraron que la población beneficiada es $100 \%$ estrato 1 , el uso de la vivienda es residencial, la actividad principal es la agricultura y ganadería, la fuente de agua para el consumo humano el $93 \%$ la obtienen de pozo profundo con bomba. La mayoría de las casas tienen piso de tierra o cemento, los techos de las viviendas son de zinc y otras de palma, el 30\% de las viviendas no tienen inodoro con pozo séptico, no existe alcantarillado, el $83 \%$ usaban velas y linternas para iluminación, La mayoría de las viviendas queman la basura.

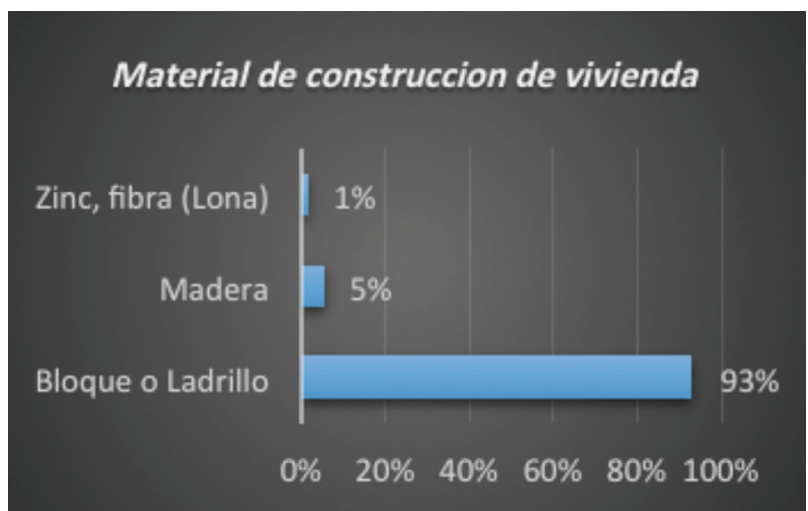

Figura 3. Material de construcción de vivienda

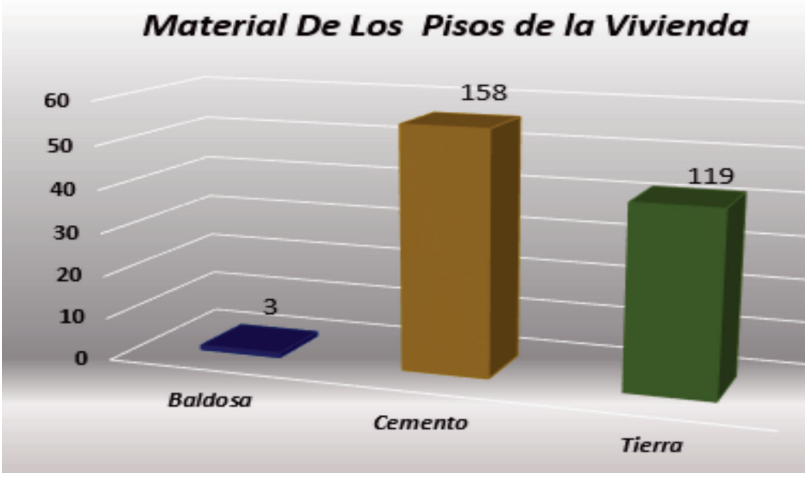

Figura 4. Material de los pisos de la vivienda

\section{Material en que esta construido el techo de la Vivienda}

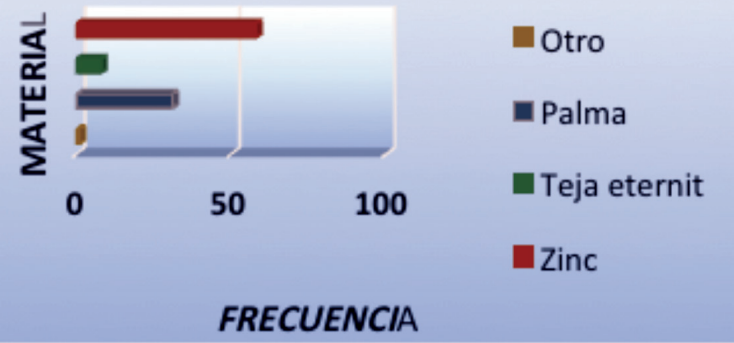

Figura 5. Material de construcción del techo de la vivienda

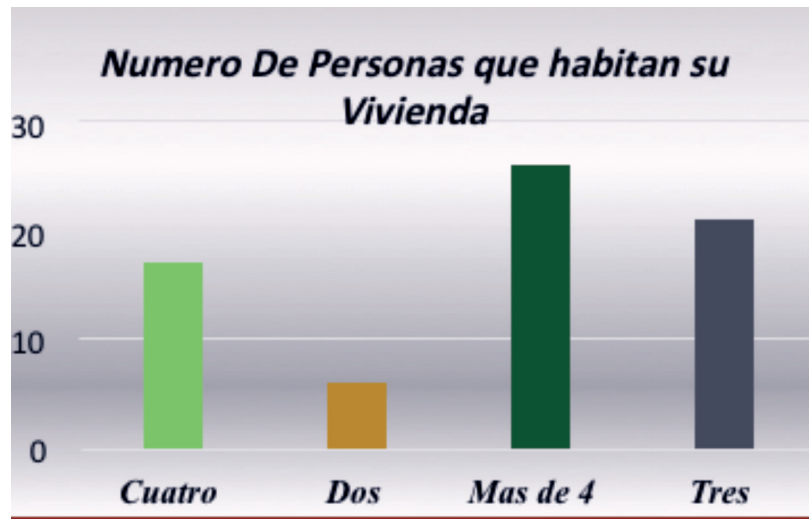

Figura 6. Número de personas por vivienda 


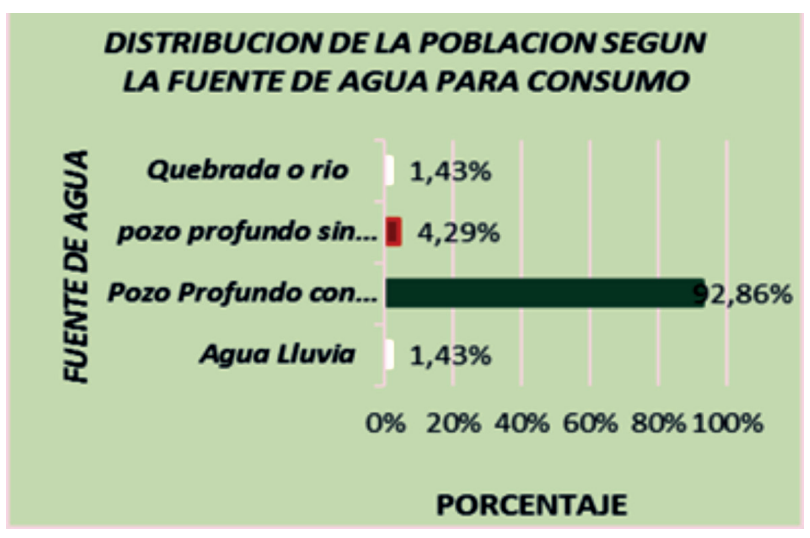

Figura 7. Fuente de agua para consumo

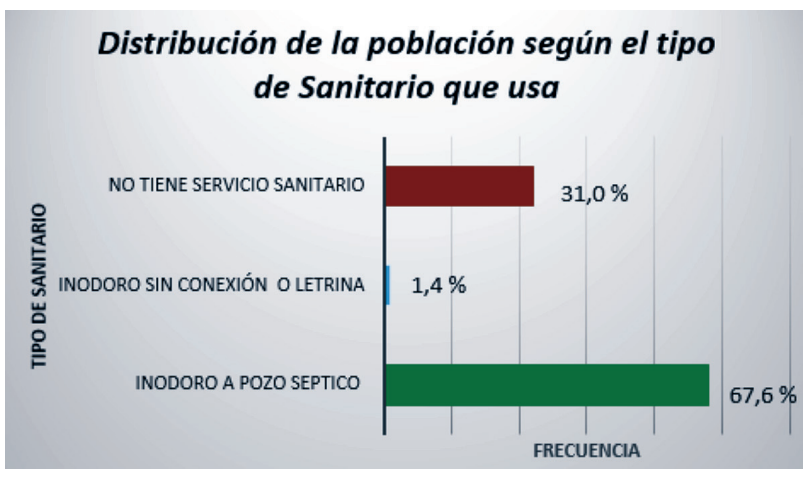

Figura 8. Tipo de sanitario de la vivienda

\section{Análisis Ambiental}

Finalmente, en lo relacionado con el aspecto ambiental se destaca que el SFV emite un $71,3 \%$ menos de GEI Gases Efecto Invernadero que los que emite el SIN, con una reducción en emisiones equivalente a $162 \mathrm{Kg} \mathrm{CO}_{2 \text { eq }}$ / año por cada usuario

\section{FUENTE DE ILUMINACION PARA LAS VIVIENDAS}

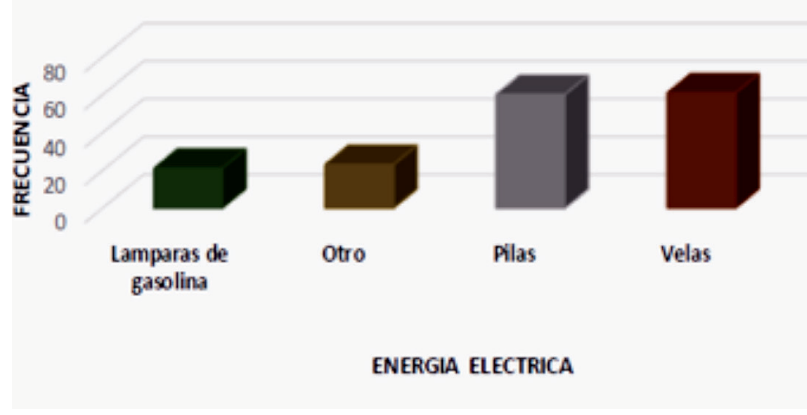

Figura 9. Fuente de iluminación de la vivienda

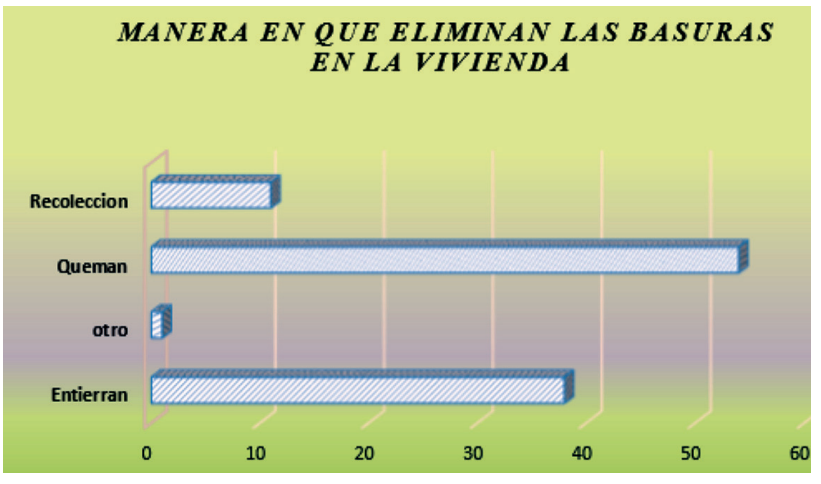

Figura 10. Eliminación de basuras de la vivienda

Tabla 2. Emisiones de Gases Efecto Invernadero

\begin{tabular}{l|c|c|c}
\hline \multicolumn{1}{c}{ Tipo de Fuente } & $\begin{array}{c}\text { Energía Producida SFV } \\
\text { KWh/año }\end{array}$ & $\mathbf{g ~ C O}_{\text {2eq }} / \mathbf{K W h}$ & $\mathbf{~ k g ~ C O}_{\text {2eq }} /$ /año \\
\hline SIN Colombia & 1251 & 195 & 244 \\
\hline Solar FV $1110 \mathrm{Wp}$ & 1251 & 56 & 70 \\
\hline Reducción emisión & & & 174 \\
\hline \% Reducción emisión & & & $71.3 \%$ \\
\hline
\end{tabular}




\section{Conclusiones y recomendaciones}

Se pudo evidenciar que la potencia eléctrica instalada a través de este sistema solar fotovoltaico cubre las necesidades energéticas de una vivienda unifamiliar con grandes ventajas en rentabilidad económica e impacto ambiental. La población beneficiada recibió capacitación en el uso y manejo de este sistema solar fotovoltaico para vivienda aislada a través de instructores del SENA, quienes orientaron de forma teórica y práctica a los habitantes. La ubicación geográfica de Hato Corozal y sus condiciones climáticas son favorables para el buen funcionamiento y eficiencia de los paneles solares.

Dada la actual problemática mundial, derivada del calentamiento global y el cambio climático por el uso exuberante de los combustibles fósiles, la energía solar fotovoltaica es una gran alternativa como fuente de suministro energético cuyo uso debería justificarse por encima de consideraciones económicas. Sin embargo, gracias al desarrollo tecnológico que ha experimentado el sector solar, hoy día está por encima de cualquier otro tipo de energía renovable o de generación eléctrica.

En cuanto a las energías renovables la mayoría no tenía conocimiento en el tema, recibieron capacitación de instructores del SENA. Estuvieron muy atentos en la instalación y puesta en funcionamiento del SFV, recibieron a gusto y se comprometieron a darle un buen uso y seguir las instrucciones de los instructores y de los instaladores.

Para el diseño del sistema se debe conocer la radiación solar a través de mapas de la UPME, Establecer las necesidades energéticas de la comunidad o de la familia, seleccionar y cotizar los componentes del sistema. Para la instalación se debe verificar la orientación e inclinación de los paneles más eficiente, consultar a técnicos y especialistas en el tema. Analizar técnicamente la producción energética y a través de capacitación, procurar valorar y hacer un buen uso del sistema.
Este sistema de energía requiere de inversiones que pueden ser asumidas por sectores económicos fuertes como alcaldías municipales, ONGS, cooperación internacional y empresarios.

\section{Literatura citada}

1. Aguer M., Jutglar L. \& Miranda A. (2004). El ahorro energético, Ediciones Díaz de Santos, Estudios de viabilidad económica. España.

2. Comisión Económica para América Latina y el Caribe CEPAL, Naciones Unidas (2009). Contribución de los servicios energéticos a los objetivos de desarrollo del milenio y a la mitigación de la pobreza en América Latina y el Caribe, Santiago de Chile.

3. Concejo municipal de Yopal. (2012). Plan de desarrollo "Yopal con sentido social 2012-2015", Yopal, Casanare, Colombia.

4. Cherny, J. (2004). Renewable energy for sustainable rural livelihoods resurl technical report, Imperial College London.

5. Domenech, L. B. (2013). Metodología para el diseño de sistemas de electrificación autónomos para comunidades rurales, (Tesis doctoral), Universidad Politécnica de Cataluña, Barcelona.

6. PNUD. (2012). Energía asequible y sostenible. Recuperado de http://www.undp.org/content/undp/es/home/sdgoverview/post-2015-development-agenda/goal-7.html

7. Empresa de Energía de Casanare. (2003). Informe ejecutivo de gestión. Recuperado de: http://www.superservicios.gov.co/content/download/2003/21285/ version/1/file/(2013)+INFORME+EJECUTIVO+DE++D $\mathrm{E}+\mathrm{GESTION}+\mathrm{EMPRESA}+\mathrm{DE}+\mathrm{ENERGIA+DE+CASA}$ NARE+SA+ESP.pdf.

8. IEA, UNDP, UNIDO. (s.f.). worldenergyoutlook.org. Recuperado de: http://www.worldenergyoutlook.org/media/ weowebsite/energydevelopment/weo2010_poverty.pdf

9. Garcés, P. (2013). Organización Latinoamericana de Energía (OLADE), Energía Sostenible, Perspectiva Regional: Centroamérica - América Latina y el Caribe, Seminario Regional de Capacitación para América Central (SICA).

10. Gasquet, H. L. (2004). Conversión de la luz solar en energía eléctrica, manual teórico y práctico sobre los sistemas fotovoltaicos. Solartronic, S.A. de C.V. Departamento de Información Científica y Técnica Cuernavaca, Morelos, México.

11. Javadi, F.S. Rismanchi B. Sarraf M. Afshar O. Saidur R. Ping H.W. \& Rahim N.A. (2013). Global policy of rural electrification. Energy for Sustainable Development.. Renewable and Sustainable Energy Reviews. Elsevier 402 - 416. 324-329.

12. Kebir, N. \& Neumann, K. (2011) Research needs for meeting the challenge of decentralized energy supply in developing countries. 
13. Miguel A. (2014). Veredas de Hato Corozal tendrán electricidad a partir de radiación solar. Recuperado de: http://prensalibrecasanare.com/casanare/13662veredas-de-hato-corozal-tendrbn-electricidad-a-partirde-radiaciun-solar.html

14. Modi V, McDade S, Lallement D. \& Saghir J. (2005). Energy and the Millennium Development Goals. Energy Sector Management Assistance Programme, United Nations Development Programme, UN Millennium Project, and World Bank.

15. Pinto, F. (2004). Energías renovables y desarrollo sostenible en zonas rurales de colombia. el caso de la vereda carrizal en sutamarchán. Cuadernos de desarrollo Rural.

16. Ministerio de Minas y Energía (2013). Plan indicativo de expansión de cobertura de energía eléctrica (2013
- 2017), República de Colombia, Unidad de Planeación Minero Energética - UPME.

17. Richard A. \& Itusaca C. (2010) Cálculo de la energía generada por un sistema fotovoltaico conectado a red a 3800 msnm, Universidad Nacional del Altiplano Puno, IV Conferencia Latino Americana de Energía Solar (IV ISES_CLA) y XVII Simposio Peruano de Energía Solar (XVII-SPES). Cusco.

18. Sánchez, C. (2010). Sistemas de energía solar fotovoltaica aplicados a viviendas residenciales en entorno urbano. Universidad Internacional de Andalucía, ISBN 978-84-693-3769-1, España.

19. World Energy Trilemma WET. (2012). El trilemma energético. Recuperado de https://www.worldenergy.org/wp-content/uploads/2012/12/TrilemaEnerg\%C3\%A9tico-2012-Espanol.pdf
Conflicto de Intereses

Los autores declaran no tener ningún conflicto de intereses

Recibido: julio 25 de 2016 Aceptado: agosto 30 de 2016 